\title{
Energy-Based Collaborative Source Localization Using Acoustic Microsensor Array
}

\author{
Dan Li \\ Department of Electrical and Computer Engineering, University of Wisconsin-Madison, 1415 Engineering Drive, \\ Madison, WI 53706-1691, USA \\ Email:dli@cae.wisc.edu \\ Yu Hen Hu \\ Department of Electrical and Computer Engineering, University of Wisconsin-Madison, 1415 Engineering Drive, \\ Madison, WI 53706-1691, USA \\ Email: hu@engr.wisc.edu
}

Received 9 January 2002 and in revised form 13 October 2002

\begin{abstract}
A novel sensor network source localization method based on acoustic energy measurements is presented. This method makes use of the characteristics that the acoustic energy decays inversely with respect to the square of distance from the source. By comparing energy readings measured at surrounding acoustic sensors, the source location during that time interval can be accurately estimated as the intersection of multiple hyperspheres. Theoretical bounds on the number of sensors required to yield unique solution are derived. Extensive simulations have been conducted to characterize the performance of this method under various parameter perturbations and noise conditions. Potential advantages of this approach include low intersensor communication requirement, robustness with respect to parameter perturbations and measurement noise, and low-complexity implementation.
\end{abstract}

Keywords and phrases: target localization, source localization, acoustic sensors, collaborative signal processing, energy-based, sensor network.

\section{INTRODUCTION}

Distributed networks of low-cost microsensors with signal processing and wireless communication capabilities have a variety of applications $[1,2]$. Examples include under water acoustics, battlefield surveillance, electronic warfare, geophysics, seismic remote sensing, and environmental monitoring. Such sensor networks are often designed to perform tasks such as detection, classification, localization, and tracking of one or more targets in the sensor field. These sensors are typically battery-powered and have limited wireless communication bandwidth. Therefore, efficient collaborative signal processing algorithms that consume less energy for computation and communication are needed.

An important collaborative signal processing task is source localization. The objective is to estimate the positions of a moving target within a sensor field that is monitored by a sensor network. This may be accomplished by (a) measuring the acoustic, seismic, or thermal signatures emitted from the source-the moving target, at each sensor node of the network; and then (b) analyzing the collected source signatures collaboratively among different sensor modalities and different sensor nodes. In this paper, our focus will be on collaborative source localization based on acoustic signatures.
Source localization based on acoustic signature has broad applications: in sonar signal processing, the focus is on locating under-water acoustic sources using an array of hydrophones [3, 4]. Microphone arrays have been used to localize and track human speakers in an indoor room environment for the purpose of video conferencing $[5,6,7,8]$. When a sensor network is deployed in an open field, the sound emitted from a moving vehicle can be used to track the locations of the vehicle $[9,10]$.

To enable acoustic source localization, two approaches have been developed: for a coherent, narrowband source, the phase difference measured at receiving sensors can be exploited to estimate the bearing direction of the source [11]. For broadband source, time-delayed estimation has been quite popular $[6,9,10,12,13,14]$.

In this paper, we present a novel approach to estimate the acoustic source location based on acoustic energy measured at individual sensors. It is known that in free space, acoustic energy decays at a rate that is inversely proportional to the distance from the source [15]. Given simultaneous measurements of acoustic energy of an omnidirectional point source at known sensor locations, our goal is to infer the source location based on these readings. 
While the basic principle of this proposed approach is simple, to achieve reasonable performance in an outdoor wireless sensor network environment, the following number of practical challenges must be overcome.

(i) In an indoor environment, sound propagation may be interfered by room reverberation [16] and echoes. Similar effects may also occur in an outdoor environment when man-made walls or natural rocky hills are present within the sensor field.

(ii) In an outdoor environment, the sound propagation may be affected by wind direction $[17,18]$ and presence of dense vegetation [19].

(iii) The sensor locations may not be accurately measured.

(iv) The acoustic energy emission may be directional. For example, the engine sound of a vehicle may be stronger on the side where the engine locates. The physical size of the acoustic source may be too large to be adequately modeled as a point source.

(v) In an outdoor environment, strong background noise including wind gust may be encountered during operation. In addition, the gain of individual microphones will need to be calibrated to yield consistent acoustic energy readings.

(vi) If there are two or more closely spaced acoustic sources, their corresponding acoustic signals may interfere each other, rendering the energy decay model infeasible.

In this paper, we first propose a simple, yet powerful acoustic energy decay model. A simple field experiment result is reported to justify the feasibility of this model for the sensor network application. A maximum-likelihood estimation problem is formulated to solve the location of a single acoustic source within the sensor field. This is solved by finding the intersection of a set of hyperspheres. Each hypersphere specifies the likelihood of the source location based on the acoustic energy readings of a pair of sensors. Intersecting many hyperspheres formed by a group of sensors within the sensor field will yield the source location. This is formulated as a nonlinear optimization problem of which fast optimization search algorithms are available.

This proposed energy-based localization (EBL) method will potentially give accurate results at regular time interval, and will be robust with respect to parameter perturbations. It requires relatively few computations and consumes little communication bandwidth, and therefore is suitable for low power distributed wireless sensor network applications.

This paper is organized as follows. In Section 2, we review several existing source localization algorithms. In Section 3, an energy decay model of sensor signal readings is provided. An outdoor experiment to validate this model is also outlined. The development of the EBL algorithm is specified in Section 4, where we also elaborate the notion of the target location circles/spheres and some properties associated with them. A variety of search algorithms for optimizing the cost function are also proposed in this section. In Section 5, simulation is performed with the aim of studying the effect of different factors on the accuracy and precision of the location estimate. A comparison of different search al-

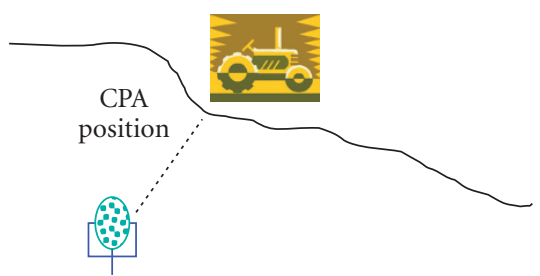

(a)

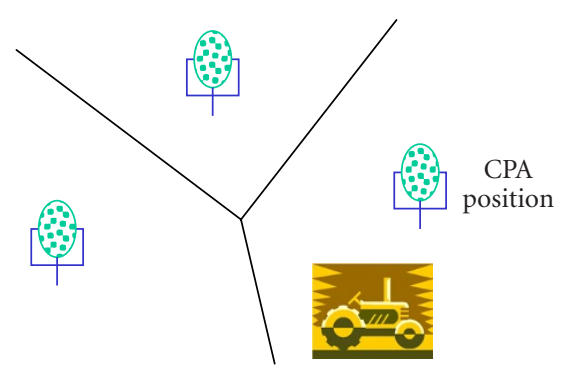

(b)

FigURE 1: Illustration of CPA-based localization (a) 1D CPA localization, (b) 2D CPA localization.

gorithms applied on our energy-based localizer is also reported.

\section{EXISTING SOURCE LOCALIZATION METHODS}

In a sensor network, a number of methods can be used to locate and track a particular moving target. Some existing methods are reviewed in this section.

\subsection{CPA-based localization method}

In its original definition, a CPA (closest point of approach) point refers to the positions of two dynamically moving objects at which they reach their closest possible distance (see, http://www.geometryalgorithms.com/Archive/ algorithm_0106/algorithm_0106.htm). In a sensor network application, a CPA position is a point on the trajectory of a moving target that is closest with respect to a stationary sensor node. Refer to Figure 1, using CPA point to estimate the target location can be accomplished in two different ways.

(i) One-dimensional CPA localization: if a target is moving along a road with known coordinates, the CPA point with respect to a given sensor node is a coordinate on this road that is closest to this observing sensor. Given the sensor coordinate and the road coordinates, this CPA point can be precomputed prior to operation. Assuming that the signal intensity will reach maximum when a target is in the closest position, the time instant, when the target is on the CPA point, can be estimated from the time series observed at the sensor. $\mathrm{Al}$ ternatively, the 1D CPA detection can be realized using a tripped-wire style sensing modality, such as a polarized infrared (PIR) sensor. 
(ii) Two-dimensional localization: in a two-dimensional sensor field, if the coordinate of the target trajectory is not known in advance, the target position cannot be precomputed. However, if the single intensity measured at neighboring sensors during the same time interval can be compared, the sensor which measures the highest acoustic signal intensity should be the one that is closest to the target. Then the location of that sensor may be used as an estimate of the target location. This is equivalent to the partition of the sensor field into $N$ Voronoi regions where $N$ is the number of sensors. If the target is in $i$ th region, the corresponding ith sensor's location will be used as an estimated location of the target.

To use the CPA style localization method, it is desirable that sufficient number of sensors are deployed within a given sensor field. Otherwise, the accuracy of the localization results may be too coarse to yield less accurate results.

\subsection{Target localization based on time delay estimation}

Sound signal travels at a finite speed. The same signal reaches sensors at different locations with different amount of delays. Denote $v$ to be the source signal propagation speed $r_{s}$ and $r_{i}$, respectively, to be the target location and ith sensor's location, and $t_{i}$ to be the time lags experienced at $i$ th sensor. Then the time delay between the source and received signal at sensor $i$ is $t_{i}=\left|r_{s}-r_{i}\right| / v+n_{i}$, where $n_{i}$ is used to model a random noise due to measurement error. While the absolute value of $t_{i}$ cannot be measured without knowing the source location $r_{s}$, the relative time delay measured with respect to a reference sensor $r_{0}$ can be measured as

$$
v\left(t_{i}-t_{0}\right)=v \tilde{t}_{i}=\left|r_{s}-r_{i}\right|-\left|r_{s}-r_{0}\right|+\tilde{n}_{i}
$$

Given $N+1$ sensors, $N$ equations like (1) can be formulated. Then, one may estimate the unknown parameters $v$ and $r_{s}$ using maximum likelihood estimation $[6,10,14,20]$.

Alternatively, (1) can be expressed as

$$
\begin{aligned}
{\left[-2\left(r_{i}-r_{0}\right)^{T}\right.} & \left.\left|r_{i}-r_{0}\right|^{2}-2\left(t_{i}-t_{0}\right)\right] \mathbf{x} \\
& =\mathbf{a}_{i}^{T} \mathbf{x}=\left(t_{i}-t_{0}\right)^{2}=b_{i}, \quad 1 \leq i \leq N,
\end{aligned}
$$

where $\mathbf{x}=\left[\left(r_{s}-r_{0}\right)^{T} 1 /|v|^{2}\left|r_{s}-r_{0}\right| / v\right]^{T}$ is a $(d+2) \times 1$ vector with $d$ being the dimension of the sensor and target location vector. Note that elements of $\mathbf{x}$ are interdependent. With $N+1(>d+2)$ sensors, the target location can be found by solving a constrained quadratic optimization problem: find $\mathbf{x}$ to minimize $C=\|\mathbf{A} \mathbf{x}-\mathbf{b}\|^{2}$ subject to

$$
x_{d+1} \cdot\left(\sum_{i=1}^{d} x_{i}^{2}\right)=x_{d+2}^{2},
$$

where $\mathbf{A}=\left[\mathbf{a}_{1} \mathbf{a}_{2} \cdots \mathbf{a}_{N}\right]^{T}$ and $\mathbf{b}=\left[b_{1} b_{2} \cdots b_{N}\right]^{T}$. The constraint described by (3) is due to the interdependent relations between elements of the $\mathbf{x}$ vector. The target location can be estimated as $r_{s}=r_{0}+\left[x_{1} \cdots x_{d}\right]^{T}$, and the propagation speed can be solved simultaneously as $v=1 / \sqrt{x_{d+1}}$. If constraint (3) is ignored, one would need only to solve an overdetermined linear system $\mathbf{A x}=\mathbf{b}$ using the least square method [9]. This method has also been refined using iterative improvement method and the Cramér-Rao bound of parameter estimation error has been derived [20]. Time-delayed estimation-based source localization methods require accurate estimation of time delays between different sensor nodes. To measure the relative time delay, acoustic signatures extracted from individual sensor node must be compared. In the extreme case, this will require the transmission of the raw time series data that may consume too much wireless channel bandwidth. Alternative approaches include cross-spectrum [8] and range difference method [21].

\section{ENERGY-BASED COLLABORATIVE SOURCE LOCALIZATION ALGORITHM}

Energy-based source localization is motivated by a simple observation that the sound level decreases when the distance between sound source and the listener becomes large. By modeling the relation between sound level (energy) and distance from the sound source, one may estimate the source location using multiple energy readings at different known sensor locations.

\subsection{An energy decay model of sensor signal readings}

When the sound is propagating through the air, it is known that [15] the acoustic energy emitted omnidirectionally from a sound source will attenuate at a rate that is inversely proportional to the square of the distance. To verify whether this relation holds in a wireless sensor network system with a sound generated by an engine, we conducted a field experiment. In the absence of the adverse conditions laid out in the introduction above, the experiment data confirms that such an energy decay model is adequate. Details about this experiment will be reported in Section 3.2.

Let there be $N$ sensors deployed in a sensor field in which a target emits omnidirectional acoustic signals from a point source. The signal energy measured on the $i$ th sensor over a time interval $t$, denoted by $y_{i}(t)$, can be expressed as follows:

$$
y_{i}(t)=g_{i} \cdot \frac{s\left(t-t_{i}\right)}{\left|r\left(t-t_{i}\right)-r_{i}\right|^{\alpha}}+\varepsilon_{i}(t) .
$$

In (4), $t_{i}$ is the time delay for the sound signal propagates from the target (acoustic source) to the $i$ th sensor, $s(t)$ is a scalar denoting the energy emitted by the target during time interval $t ; r(t)$ is a $d \times 1$ vector denoting the coordinates of the target during time interval $t ; r_{i}$ is a $d \times 1$ vector denoting the Cartesian coordinates of the $i$ th stationary sensor; $g_{i}$ is the gain factor of the $i$ th acoustic sensor; $\alpha(\approx 2)$ is an energy decay factor, and $\varepsilon_{i}(t)$ is the cumulative effects of the modeling error of the parameters $g_{i}, r_{i}$, and $\alpha$ and the additive observation noise of $y_{i}(t)$. In general, during each time duration $t$, 
many time samples are used to calculate one energy reading $y_{i}(t)$ for sensor $i$. Based on the central limit theorem, $\varepsilon_{i}(t)$ can be approximated well using a normal distribution with a positive mean value, denoted by, say, $\mu_{i}(>0)$, that is no less than the standard deviation (STD) of the background measurement noise during that time interval. The STD of $\varepsilon_{i}(t)$ may also be empirically determined.

\subsection{Experiment that validates the acoustic energy decay model}

To validate the model described in (4), we conducted a field experiment. We used a lawn mower at stationary position as our acoustic energy source. Two sensor nodes with acoustic microphone used in a DARPA SensIT project are placed at different distances $(5 \mathrm{~m}, 10 \mathrm{~m}, 15 \mathrm{~m}, 20 \mathrm{~m}, 25 \mathrm{~m}, 30 \mathrm{~m})$ away from the energy source. The microphones are placed at about $50 \mathrm{~cm}$ above the ground and face the energy source. The weather is clear with gentle breeze, and the temperature is about $24^{\circ} \mathrm{C}$.

The time series of both the acoustic sensors was recorded at a sampling rate of $4960.32 \mathrm{~Hz}$. Then the energy readings were computed offline as the moving average (over a 0.5 second sliding window) of the squared magnitude of the time series. These energy readings then were fitted to an exponential curve to determine the decaying exponent $\alpha$, as shown in Figure 2.

For both acoustic sensors, within the 30-meter range, the acoustic energy decay exponents are $\alpha=2.1147$ (with mean square error 0.054374 ) and $\alpha=2.0818$ (with mean square error 0.016167$)$, respectively. This validates the hypothesis that the acoustic energy decreases approximately as the inverse of the square of the source sensor distance.

We here assume $\alpha$ to be constant, which is valid if the sound reverberation can be ignored and the propagation medium (air) is roughly homogenous (i.e., no gusty wind) during the process of experiment.

\subsection{Maximum likelihood parameter estimation}

Assume that $\varepsilon_{i}(t)$ in (4) are independent, identically distributed (i.i.d.) normal random variables with known mean $\mu_{i}(>0)$ and known variance $\sigma_{i}^{2}$, then each $y_{i}(t)$ will be an i.i.d. conditional normal random variable with a probability density function $N\left(g_{i} s(t) /\left|r(t)-r_{i}\right|^{\alpha}+\mu_{i}, \sigma_{i}^{2}\right)$. We also assume that the time delay discrepancies among sensors are negligible, that is, $t_{i}=0$. Then, the likelihood function or, equivalently, the conditional joint probability can be expressed as follows:

$$
\begin{aligned}
& \ell(s(t), r(t)) \\
& \quad=f\left(y_{0}(t), \ldots, y_{N-1}(t) \mid \sigma^{2},\{s(t), r(t)\}\right) \\
& \quad \propto \exp \left\{-\frac{1}{2} \sum_{i=0}^{N-1}\left\{\frac{\left[y_{i}(t)-\mu_{i}-g_{i} s(t) /\left|r(t)-r_{i}\right|^{\alpha}\right]^{2}}{\sigma_{i}^{2}}\right\}\right\} .
\end{aligned}
$$

The objective of the maximum likelihood estimation is to find the source energy reading and the source locations
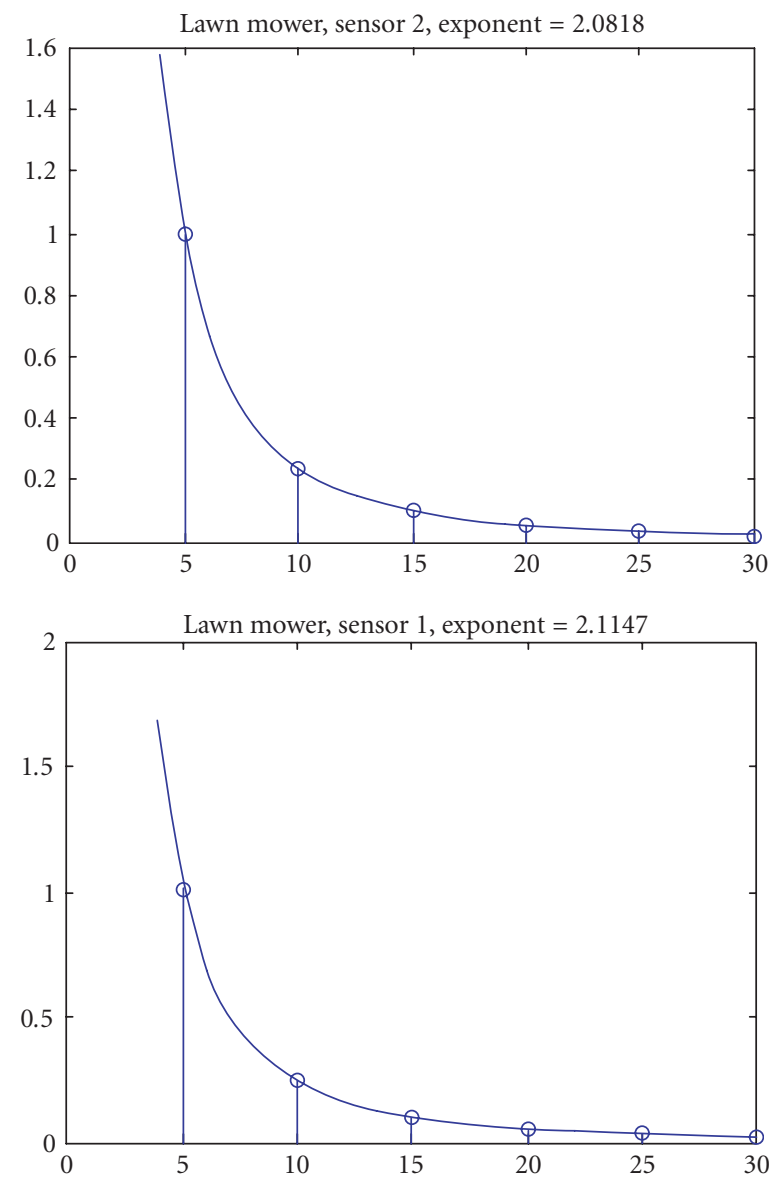

FIgURE 2: Acoustic energy decay profile of the lawn mower and the exponential curve fitting.

$\{s(t), r(t)\}$ to maximize the likelihood function. Since we assume that the mean $\mu_{i}$ and the variance $\sigma_{i}^{2}$ of $\varepsilon_{i}(t)$ are known, this is equivalent to minimizing the following log-likelihood function:

$$
L(s(t), r(t)) \propto \sum_{i=0}^{N-1}\left\{\frac{\left[y_{i}(t)-\mu_{i}-g_{i} s(t) /\left|r(t)-r_{i}\right|^{\alpha}\right]^{2}}{\sigma_{i}^{2}}\right\}
$$

Given $\left\{y_{i}(t), g_{i}, r_{i}, \mu_{i}, \sigma_{i}^{2} ; 0 \leq i \leq N-1\right\}$ and $\alpha$, the goal is to find $s(t)$ and $r(t)$ to minimize $L$ in (6). This can be accomplished using a standard nonlinear optimization method such as the Nelder-Mead simplex (direct search) method implemented in the optimization package in Matlab.

\subsection{Energy ratio and target location hypersphere}

In the above formulation, we solve for both the source location $r(t)$ and source energy $s(t)$. In this section, we present an alternative approach that is independent of the source energy $s(t)$. This is accomplished by taking ratios of the energy readings of a pair of sensors in the noise-free case to cancel out $s(t)$. We refer to this approach as the energy ratio formulation. 


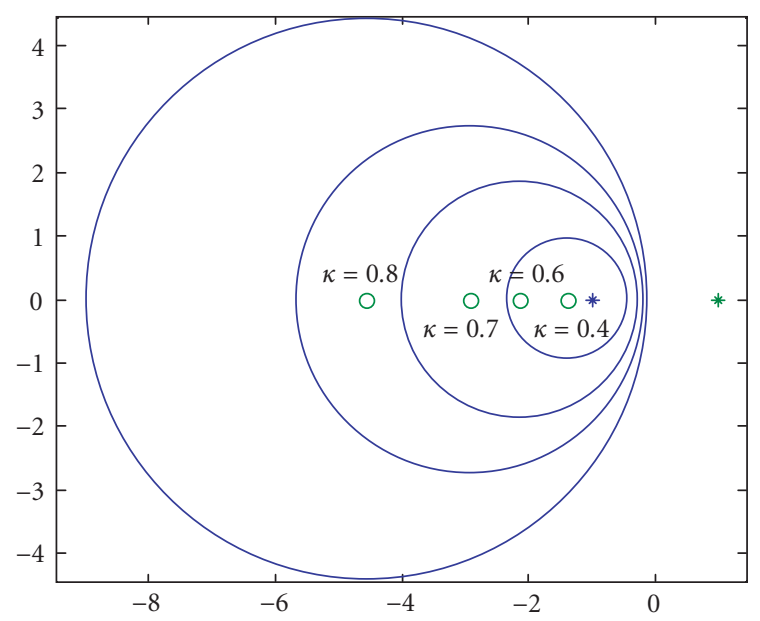

FIgURE 3: Two sensors are located at $(-1,0)$ and $(1,0)$. Four $\kappa$ values are used $0.4,0.6,0.7$, and 0.8 . The corresponding target location circles and their centers are also shown.

Approximating the additive noise term $\varepsilon_{i}(t)$ in (4) by its mean value $\mu_{i}$, we can compute the energy ratio $\kappa_{i j}$ of the $i$ th and the $j$ th sensors as follows:

$$
\kappa_{i j}:=\left(\frac{\left(y_{i}(t)-\mu_{i}\right) /\left(y_{j}(t)-\mu_{j}\right)}{g_{i}(t) / g_{j}(t)}\right)^{-1 / \alpha}=\frac{\left|r(t)-r_{i}\right|}{\left|r(t)-r_{j}\right|} .
$$

Note that for $0<\kappa_{i j} \neq 1$, all the possible source coordinates $r(t)$ that satisfy (7) must reside on a $d$-dimensional hypersphere described by the equation

$$
\left|r(t)-c_{i j}\right|^{2}=\rho_{i j}^{2}
$$

where the center $c_{i j}$ and the radius $\rho_{i j}$ of this hypersphere associated with sensor $i$ and $j$ are given by

$$
c_{i j}=\frac{r_{i}-\kappa_{i j}^{2} r_{j}}{1-\kappa_{i j}^{2}}, \quad \rho_{i j}=\frac{\kappa_{i j}\left|r_{i}-r_{j}\right|}{1-\kappa_{i j}^{2}} .
$$

For convenience, we will call this hypersphere a target location hypersphere. When $d=2$, such a hypersphere is a circle. When $d=3$, it is a sphere. In Figure 3, several examples corresponding to $d=2$ and $\kappa_{i j}<1$ are illustrated. As $\kappa_{i j}$ increases, that is, as $y_{j}(t) / g_{j}(t) \rightarrow y_{i}(t) / g_{i}(t)$, the center of the circle moves away from the sensors, and the radius increases.

In the limiting case when $\kappa_{i j} \rightarrow 1$, the solution of (7) form a hyperplane between $r_{i}$ and $r_{j}$

$$
r(t) \cdot\left(r_{i}-r_{j}\right)=\frac{\left|r_{i}\right|^{2}-\left|r_{j}\right|^{2}}{2} \quad \text { or equivalently, }
$$

where

$$
\gamma_{i j}=r_{i}-r_{j}, \quad \xi_{i j}=\frac{\left|r_{i}\right|^{2}-\left|R_{j}\right|^{2}}{2} .
$$

So far, we have established that using the ratio of energy readings at a pair of sensors, the potential target location can be restricted to a hypersphere whose center and radius are functions of the energy ratio and the two sensor locations. If more sensors are used, more hyperspheres can be determined. If all the sensors that receive the signal from the same target are used, the corresponding target location hyperspheres must intersect at a particular point that corresponds to the source location. This is the basic idea of the energy-based source localization. Note that since the source energy is cancelled during the energy ratio computation, this method will not be affected even if the source energy levels vary dramatically between successive energy integration time intervals.

\subsection{Single target localization using multiple energy ratios and multiple sensors}

Suppose that $N$ acoustic sensors detected the source signal emitted from a target during the same time intervals, $N(N-1) / 2$ pairs of energy ratios can be computed. Based on $M(\leq N(N-1) / 2)$ these sensor energy ratios, our objective is to estimate the target location $r(t)$ during that time interval. Using a least square criterion, this problem leads to a nonlinear least square optimization problem where the cost function is defined as

$$
\begin{gathered}
J(r)=\sum_{m=1}^{M_{1}}||\left|r-c_{m}\right|\left|-\rho_{m}\right|^{2}+\sum_{n=1}^{M_{2}}\left|\gamma_{n}^{T} r-\xi_{n}\right|^{2}, \\
M_{1}+M_{2}=M,
\end{gathered}
$$

where $m$ and $n$ are indices of the energy ratios computed between different pairs of sensor energy readings, $M_{1}$ is the number of hyperspheres, and $M_{2}$ is the number of hyperplanes. In practice, when $\left|1-\kappa_{i j}^{2}\right|$ becomes too small, it may cause numerical problem when evaluating $r$ and $\rho$ using (9). In this case, the hyperplane equation (10) should be used instead. In our simulation, a value of $10^{-3}$ was set as the threshold to switch between these two type of error terms.

Note that if two sensors are both close to the target, their energy readings have higher SNRs. Therefore, the energy ratio $\kappa_{i j}$ computed from these energy readings will be more reliable than that computed from a pair of sensors far away from the target. Using the energy decay model, we may use the relative magnitudes of energy readings as an indication of the target-sensor distance. As such, the error term in (12) that correspond to sensors with higher-energy readings should be given more weight than sensors that have lowerenergy readings.

Statistically, to employ the least square formulation in (12), one must assume that both the hypersphere estimation error $\left\|r-c_{m}\right\|-\rho_{m}$ and the hyperplane estimation error $\gamma_{n}^{T} r-\xi_{n}$ are linear, independent Gaussian random variables with zero mean and identical variance. Obviously, such an assumption may not be true in practice and hence may cause some performance degradation.

The cost function in (12) is nonlinear with respect to the source location vector $r$. In this work, we experimented with three nonlinear optimization methods to solve for $r$. 
(a) Exhaustive search over grid points within a predefined search region in the sensor field. This approach is the most time consuming, yet most simple to implement. The grid size determines the accuracy of the results.

(b) Multiresolution search. First a coarse-grained exhaustive search is conducted to identify likely source locations. Then a detailed fine-grained search is performed to refine the localization estimate.

(c) Gradient-based steepest descent search method. Based on an initial source location (perhaps the previously estimated position in the last time interval), say $r(0)$, perform the following iteration:

$$
r(k+1)=r(k)-\mu \nabla_{r} J(r) .
$$

The gradient of $J(\underline{r})$ can be expressed as

$$
\nabla J(\underline{r}) 2 \sum_{m=1}^{M_{1}} \frac{\underline{r}-\underline{c}_{m}}{\left\|\underline{r}-\underline{c}_{m}\right\|}\left(\left\|\underline{r}-\underline{c}_{m}\right\|-\rho_{m}\right)+2 \sum_{n=1}^{M_{2}} \gamma_{n}\left(\gamma_{n}^{T} r-\xi_{n}\right) .
$$

In addition to the above methods, other standard optimization algorithms, such as the quasi-Newton's method, conjugate gradient search algorithm, and many others can be used. For comparison purpose, in the simulation, we also apply the Nelder-Mead (direct search) method implemented in Matlab optimization toolbox to minimize $J(r)$.

In summary, there are two different methods to solve the energy-based, (single) source localization problem.

(1) Direct minimization of the nonlinear log-likelihood function $L$ as in (7). With a number of acoustic energy measurements, this method is capable of simultaneously estimating the source location $r(t)$ as well as the source energy $s(t)$, and the energy decay parameter $\alpha$.

(2) Direct minimization of the cost function defined in (12). A potential advantage of this method is that $N(N-1) / 2$ pairs of energy ratios can be used for the localization purpose rather than the $N$ energy readings used for minimizing the likelihood function.

\subsection{Unconstrained least square formulation}

Consider two hyperspheres based on (8)

$$
\left|r(t)-c_{i 0}\right|^{2}=\rho_{i 0}^{2}, \quad\left|r(t)-c_{j 0}\right|^{2}=\rho_{j 0}^{2} .
$$

They are formed from the sensor pairs $(i, 0)$ and $(j, 0)$. Subtract each side and cancel the term $|r(t)|^{2}$, we have a hyperplane equation

$$
2\left(c_{i 0}-c_{j 0}\right) r(t)=\left(c_{i 0}^{2}-\rho_{i 0}^{2}\right)-\left(c_{j 0}^{2}-\rho_{j 0}^{2}\right) .
$$

Substitute the definition in (9), the above equation is simplified to

$$
u_{i j} r(t)=\theta_{i j}
$$

which is a linear hyperplane equation with

$$
u_{i j}=\frac{2 r_{i}}{1-\kappa_{i}^{2}}-\frac{2 r_{j}}{1-\kappa_{j}^{2}}, \quad \theta_{i j}=\frac{\left|r_{i}\right|^{2}}{1-\kappa_{i}^{2}}-\frac{\left|r_{j}\right|^{2}}{1-\kappa_{j}^{2}}
$$

Then, the cost function in (12) can be replaced by a linear least square cost function

$$
j_{\text {Linear }}(r)=\sum_{m=1}^{M_{1}}\left|u_{n}^{T} r-\theta_{n}\right|^{2}+\sum_{n=1}^{M_{2}}\left|\gamma_{n}^{T} r-\xi_{n}\right|^{2} .
$$

Note that there is no constraint imposed in (19). Given the coefficients, a solution of $r$ can be found in closed form.

\section{IMPLEMENTATION CONSIDERATIONS}

\subsection{Preprocessing: node and region energy detection}

In a microsensor network, multiple acoustic sensors are deployed in a sensor field. Sensors within the same geographical region will form a group. One sensor node in a group will be designated as a manager node where the collaborative energy-based source localization will be performed.

During operation, individual sensor nodes will perform energy-based target detection algorithm. For example, a constant false alarm rate (CFAR) detection algorithm [22, 23] can be applied. Pattern classifiers may also be used to identify the type of a detected target based on its acoustic or seismic signatures.

Upon detection of a potential target, the sensor node will report the finding to the manager node in the region. If the number of detections reported by sensors within the region exceeds a predefined threshold, the manager node then decides that a target is indeed detected by the region. This implements a simple voting-based detection fusion within the region. Only after a region-wide detection is confirmed, the manager node will proceed to perform energy-based source localization. Since the energy is computed on individual nodes, there is no need to recompute the acoustic energy readings at the manager node.

\subsection{Minimum number of collaborating sensors and number of energy ratios used}

In general, given $N$ sensors, at maximal $N(N-1) / 2$ pairs of energy ratios can be computed, and equal number of target location hyperspheres (including some hyperplanes) can be determined accordingly. The target location is the unique intersection of all these target location hyperspheres if the energy readings do not contain any measurement noise.

However, many of these relationships are actually redundant. In order to uniquely identify a single target location, in this section, we want to determine (i) the constraint on the sensor location configuration; and (ii) the minimum number of sensors required in theory to arrive at a unique source location estimate. Regarding sensor location configuration, we have the following results.

Lemma 1. Denoted to be the dimensionality of the sensor coordinate $r_{i}$. If all $N$ sensors locate on a subspace with a dimension 
$d^{\prime}<d$, then the centers of every target location hyperspheres must lie within the same subspace.

Proof. From (10), since $c_{i j}$ is a linear combination of sensor coordinates $r_{i}$ and $r_{j}$, it must lie within the same subspace as $r_{i}$ and $r_{j}$. Hence this lemma is proved.

Specifically, in a 2D $(d=2)$ sensor field, if all sensors locate on a straight line, then all the centers of the corresponding target location circles must locate on the same straight line. Since circles with their centers locating on the same straight line cannot have a single point as their intersection (either no intersection, or two or more points in the intersection), it is impossible to uniquely determine the target location. The exception is when the target location is also on the same straight line. In a 3D $(d=3)$ sensor field, if all sensors locate on the same plane, then all the centers of the corresponding target location spheres must locate on the same plane as well. Since spheres with their centers locating on the same plane cannot intersect at just a single point in general, it cannot uniquely determine the target location. Similarly, the exception is when the target locates on the same plane. These observations lead to the theorem below which is stated without proof.

Theorem 1. In order to estimate a unique target location, not all the sensors should be placed on a subspace whose dimension is smaller than that of the sensor field unless the target location is restricted in the same subspace as well.

Next, we consider the question of the minimum number of sensors needed to locate a single target.

Lemma 2. Given three arbitrary placed sensors (say, 1, 2, and 3) in a 2D sensor field, the centers of every target location circles $c_{12}, c_{23}$, and $c_{31}$ must lie on the same straight line. Moreover, the corresponding three target location circles may intersect at two points if the target does not locate on the same straight line, or at exactly one point if the target does locate on the same straight line.

Proof. Performing linear combination of $c_{12}$ and $c_{23}$ in order to eliminate $r_{2}$ and using the relations $\kappa_{12} \kappa_{23} \kappa_{31}=1$, one has

$$
\begin{aligned}
\frac{1-\kappa_{12}^{2}}{\kappa_{12}^{2}} & c_{12}+\left(1-\kappa_{23}^{2}\right) c_{23} \\
= & \frac{1-\kappa_{12}^{2}}{\kappa_{12}^{2}}\left[\frac{r_{1}-\kappa_{12}^{2} r_{2}}{1-\kappa_{12}^{2}}\right] \\
& +\left(1-\kappa_{23}^{2}\right)\left[\frac{r_{2}-\kappa_{23}^{2} r_{3}}{1-\kappa_{23}^{2}}\right] \\
= & \frac{r_{1}}{\kappa_{12}^{2}}-\kappa_{23}^{2} r_{3} \\
= & \kappa_{23}^{2} \kappa_{31}^{2} r_{1}-\kappa_{23}^{2} r_{3} \\
= & -\kappa_{23}^{2}\left(1-\kappa_{31}^{2}\right)\left[\frac{r_{3}-\kappa_{31}^{2} r_{1}}{1-\kappa_{31}^{2}}\right] \\
= & -\kappa_{23}^{2}\left(1-\kappa_{31}^{2}\right) c_{31} \\
= & \frac{1-\kappa_{12}^{2} \kappa_{23}^{2}}{\kappa_{12}^{2}} c_{31} .
\end{aligned}
$$

$$
\frac{1-\kappa_{12}^{2}}{\kappa_{12}^{2}}+\left(1-\kappa_{23}^{2}\right)=\frac{1-\kappa_{12}^{2} \kappa_{23}^{2}}{\kappa_{12}^{2}} .
$$

Since $c_{31}=\beta c_{12}+(1-\beta) c_{23}, c_{12}, c_{23}$, and $c_{31}$ must lie on the same straight line, next, note that the true target location must be a point in each of the three corresponding target location circles. In addition, three circles with their centers located on the same straight line can intersect at most two points, or not to intersect at all. Hence, these three circles must intersect at exactly two points. When the target locates on the same straight line where the centers of these circles locate, the two points of their intersection collide into a single point. Hence, this lemma is proved.

Lemma 2 implies that, even though three sensors are not on the same straight line, the centers of the corresponding target location circles (or spheres) still lie on the same straight line. Using the argument in the proof of Theorem 1, clearly three sensors are insufficient to estimate a unique target location in a $2 \mathrm{D}$ sensor field. It appears that at least four sensor energy readings will be needed.

Lemma 2 addresses the 2D sensor field case. It can easily be generalized to the $3 \mathrm{D}$ sensor field case.

Lemma 3. Given four arbitrary placed sensors in a 3D sensor field, the centers of every target location spheres must lie on the same plane. Moreover, the six corresponding target location spheres may intersect at two points if the target does not locate on the same plane. Otherwise, their intersection contains exactly one point if the target also locates on the same plane.

Proof. Label these four sensors from 1 to 4 . With four sensor energy readings, six energy ratios can be computed. Using Lemma 2, we conclude that

(i) $c_{12}, c_{13}$, and $c_{23}$ must reside on the straight line $L_{a}$;

(ii) $c_{12}, c_{14}$, and $c_{24}$ must reside on the straight line $L_{b}$;

(iii) $c_{13}, c_{14}$, and $c_{34}$ must reside on the straight line $L_{c}$.

Lines $L_{a}$ and $L_{b}$ share the same point $c_{12}$. Hence, they must lie on the same plane. Line $L_{c}$ share one point to each line $L_{a}\left(c_{13}\right)$ and line $L_{b}\left(c_{14}\right)$, respectively. Therefore, $L_{c}$ must lie on the same plane as $L_{a}$ and $L_{b}$. The intersection regions between spheres with centers on $L_{a}, L_{b}$, and $L_{c}$, respectively, are circles, respectively. With three circles in a 3D space, their intersection contains at most two points. If the target also locates on the same plane, then these two points collide into one.

Lemma 2 also reveals the redundancy among different energy ratios. This critical observation can be stated as a corollary as follows.

Corollary 1. Given energy ratios $\kappa_{1 i}$ and $\kappa_{1 j}$, the energy ratio $\kappa_{i j}$ is redundant and can be removed without affecting the solution of the target location. 
Proof. Since $\kappa_{1 i} \kappa_{i j} \kappa_{j 1}=1$. Using Lemma 2, the intersection between the target location circle (sphere), corresponding to $\kappa_{i j}$ with any of the other two circles (spheres), will be identical to the intersection between the circles (spheres) corresponding to $\kappa_{1 i}$ and $\kappa_{j 1}$. Hence, the inclusion of target location circle (sphere) of $\kappa_{i j}$ does not contribute to any new information to refine the solution space. Therefore, it is redundant.

Corollary 1 naturally leads to an important result in this section.

Lemma 4. Given $K$ sensors in a sensor field, then at most $K-1$ pairs of energy ratios are independent in that the target location circles (or spheres) corresponding to remaining energy ratios do not further reduce the intersection region formed by the $K-1$ target location circles (or spheres) of those independent energy ratios.

Proof. Denote sensor \#1 as a reference sensor. Then denote $\left\{\kappa_{1 i} ; 2 \leq i \leq K\right\}$ for the set of $K-1$ independent energy ratios. Any other energy ratio $\kappa_{j k}, 2 \leq j, k \leq K, j \neq k$ will be redundant according to Corollary 1 . Thus, this lemma is proved. Note that the set of $K-1$ independent energy ratios is not unique and can be chosen differently.

Theorem 2. Using the energy-based target localization meth$o d$, at least four sensors not locating on the same straight line are required to locate a single target in a $2 D$ sensor field; and at least five sensors not all locating on the same plane are required to locate a single target in a 3D sensor field.

Proof. In a 2D sensor field, at least $3(=K-1)$ circles are needed to form a single point intersection. Thus, at least four sensor energy readings are needed. In a 3D sensor field, the intersection of two spheres is a circle. The intersection between a sphere and a circle consists of at least two points (if the intersection exists). Therefore, at least $4(=K-1)$ spheres are needed to yield a single point intersection. Thus the minimum number of sensor energy readings needed in a 3D sensor field is five.

Figure 4 shows a simulation of target localization in a 2D sensor field using four sensors and three energy ratios.

\subsection{Nonlinear optimization search parameters}

In developing nonlinear optimization methods to minimize the cost function, a few parameters must be set properly to ensure the performance of this proposed algorithm.

\subsubsection{Search area}

The region of the potential target location can often be determined in advance, based on prior information about the target, the region to be monitored, and the sensor locations. Since acoustic energy decays exponentially with respect to distance, the receptive field of an acoustic sensor (microphone) is limited. This range can be estimated based on the maximum acoustic energy the target of interests may emit,

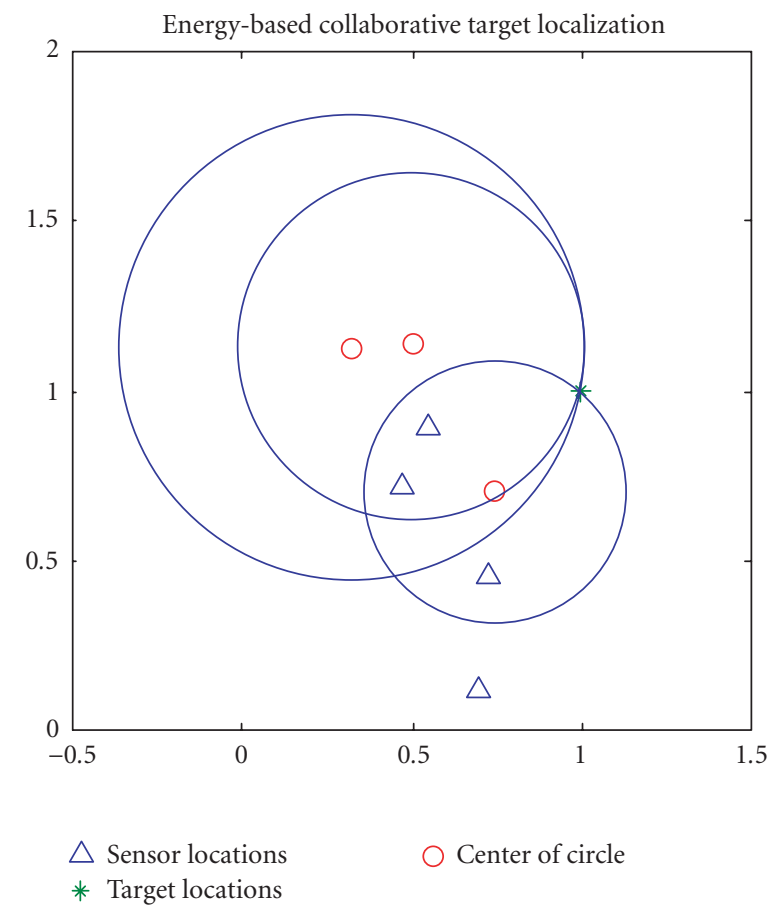

FIgURE 4: Localization of the target (star) at $(1,1)$ position using four sensors (triangle). The centers of the circles are small circles. Three circles corresponding to three independent equations are generated. These three circles intersect at the target position as predicted. Parameters used $s(t)=1, g_{i}=1$, and $\alpha=2$.

and the averaged background noise level due to wind and other natural or man-made sound. Furthermore, due to the need of collaborative region detection, a target is not considered detected unless a certain number of sensors voted positive detection. Hence the area that a target may be detected should be the intersection of a minimum number of sensors receptive fields.

If a target's movement is restrictive, such as along a road, then the search area can further be restricted to those areas where the target is allowed to move. These additional restrictions will enhance the accuracy of the source localization process.

\subsubsection{Search accuracy}

Depending on the size of the potential target and its speed, the required accuracy of localization may vary. For example, for a target with a dimension (say, length of a truck) larger than 5 meters, it would be meaningless to try to locate the target within a 1-meter grid. In addition, if the target is moving more than $10 \mathrm{~m} / \mathrm{s}$ (about $20 \mathrm{mph}$ ), and the time duration to compute one energy reading is 0.5 second, then the ambiguity regarding the actual location of the target during this time period will be at least 5 meters. In this situation, any attempt to locate the target within 5 meters will not be meaningful. Therefore, in practical implementation, one should choose appropriate accuracy measure. 


\subsubsection{Initial search location}

For gradient-based search algorithms and other greedy search algorithms, the initial search position is important. One way to select the initial target location estimate is to use the sensor location where the energy reading is the maximum among all other sensors. The heuristic is that if the sensor receives higher energy, then the true target location will be closer to that sensor. In a localize-and-track scenario, the future target location can be predicted based on its trajectory. In that case, the most likely position of the target during the present time window may be chosen as the initial search position.

\subsection{Distributive implementation}

This proposed EBL algorithm would require at least four sensor readings in order to yield a unique target location. Therefore, when implemented in a distributive sensor network, the acoustic energy readings will have to be reported to a centralized location to facilitate localization processing. To be deployed into a distributed wireless sensor network, it is desirable that a decentralized implementation of this proposed algorithm can be devised. By "decentralized," we hope to devise a computation scheme such that

(i) not all the energy readings need to be reported a centralized fusion center;

(ii) not all the computation required to evaluate the cost function (12) need to be carried out at a centralized processing center.

This can be accomplished by noting that the cost function in (12) consists of summation of independent square error terms. Given a potential target location $r$, each of the square error term can be evaluated within a sensor node as soon as it computes the $k$ value after receiving the acoustic energy reading at a neighboring sensor node. Hence, instead of transmitting the raw energy reading to the fusion center, the partially computed cost function can be transmitted instead. This way, the task of computation can be evenly distributed over individual sensors. This scheme, however, may increase the amount of internode wireless communications due to the need to pass around the partially computed cost function for each search grid.

\section{PERFORMANCE ANALYSIS}

A number of factors may affect the performance of the energy-based target localization algorithm. Due to the nonlinear nature and the complexity of the model, an analytical expression is difficult to obtain and may not reveal the respective impacts of individual factors on the overall performance. In this section, extensive simulation will be conducted to compare the effectiveness of different optimization algorithms as well as the sensitivities of the location estimates with respect to perturbations of various parameters of the model.

\subsection{Comparison of different search algorithms}

In this simulation, we compare four different optimization algorithms for a single target, acoustic source localization problem. For this purpose, 20 sensors are uniform randomly distributed in a 50 -meter by 50 -meter sensor field. The location of the target is assumed to be within this sensor field.

The objective function is the energy ratio cost function shown in (12). Two different modes are chosen to implement the cost function: in mode $0, N-1$ independent energy ratios ( $N$ : number of sensors) are used to form the cost function. In mode 1 , all possible $N(N-1) / 2$ energy ratios (with many redundant measurements) are used to form the cost function. The hypothesis is that with redundant measurements included in the cost function, it may better withstand parameter perturbations.

The following four search algorithms are implemented.

(1) Nelder-Mead (simplex) direct search (DS) algorithm: the initial source location is obtained by an exhaustive search at a grid size of 5 meters by 5 meters. For each new target location, the DS method will evaluate the cost function $11 \times 11=121$ times, and the DS search will require additional cost function evaluations.

(2) Grid-based exhaustive search (ES) with a single grid size of $1 \mathrm{~m} \times 1 \mathrm{~m}$. To estimate a target location, the ES method will evaluate the cost function $51 \times 51=2601$ times.

(3) Multiresolution (MR) search with three levels of resolution (grid sizes) at 5 meters $(5 \times), 2$ meters $(2 \times)$, and 1 meter $(1 \times)$, respectively. The number of cost function evaluations for each new target location equals to $11 \times 11+6 \times 6+3 \times 3=166$.

(4) Gradient descent (GD) search algorithm using the gradient expression shown in (13). The initial location is determined by ES at a grid size of 5 meters by 5 meters. The step size $\mu=0.05$ and maximal steps $=200$. The number of cost function evaluations for each new target location will be $11 \times 11=121$ times plus the number of gradient search steps.

Provided that the local search steps using either DS or gradient search is within 50 steps of either the DS or the GD search method, then the three search algorithms DS, $\mathrm{MR}$, and GD will require approximately the same number of cost function evaluations $(\sim 170)$. On the other hand, the ES method will require 15 times more cost function evaluations.

Four experiment configurations are designed to compare these search methods. In each configuration, a known fixed energy is emitting from the source. At each sensor, the received energy is computed according to the exponential energy decay model described in (4) with $K=1$ and $\varepsilon_{i}=0$ $(\mathrm{SNR}=\infty)$. Three parameters in this model will be perturbed in configurations \#2 to \#4, respectively, as shown in Table 1. Configuration \# 1 is the control experiment with no parameter perturbation. In configuration \#1, the energy decay constant $\alpha$ is sampled from a uniform distribution $[2-\Delta \alpha, 2+\Delta \alpha]$ with $\Delta \alpha=0.5$. In configuration \#3, each sensor's location $r$ is subject to a random perturbation of 

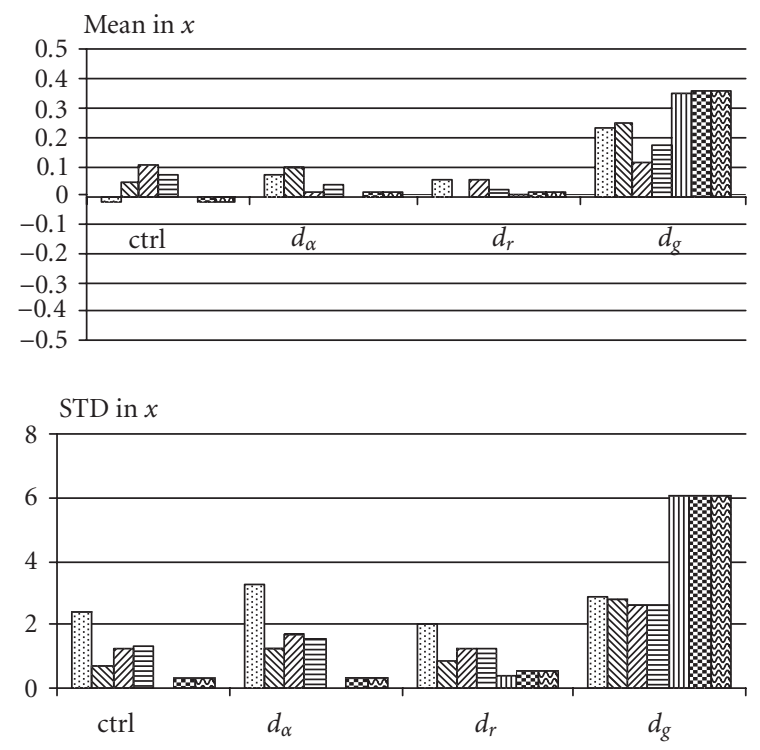
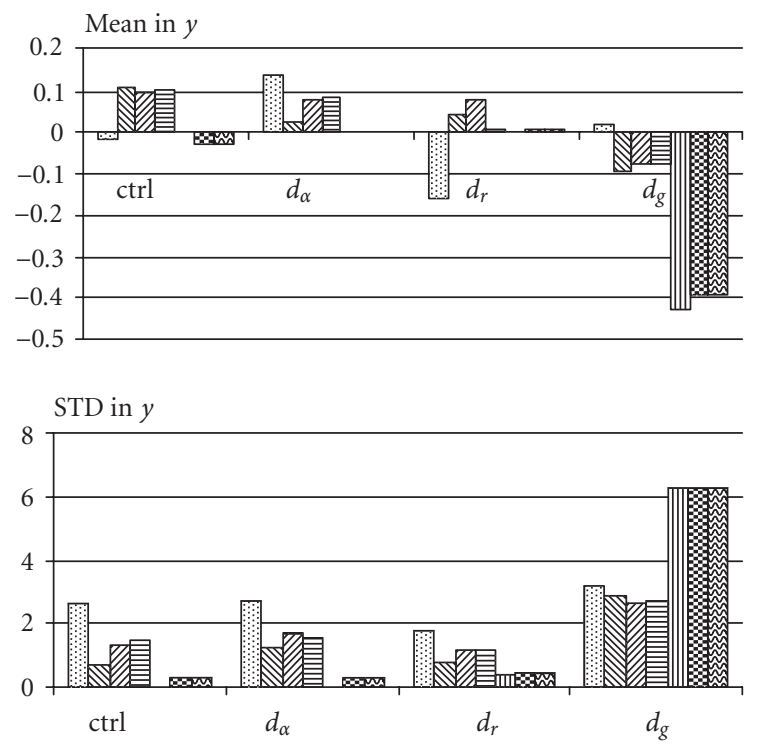

$\mathrm{MR}$, mode 0

GD, mode 0

MR, mode 1

FIGURE 5: Mean and standard deviation (STD) of target location estimation bias using different search algorithms.

TABle 1: Parameter settings for different configurations to compare four optimization search algorithms.

\begin{tabular}{clll}
\hline Configuration \# & $\Delta \alpha$ & $\Delta r$ & $\Delta g$ \\
\hline 1 & 0 & 0 & 0 \\
2 & 0.5 & 0 & 0 \\
3 & 0 & 1 & 0 \\
4 & 0 & 0 & 0.5 \\
\hline
\end{tabular}

magnitude $\pm \Delta r(= \pm 1)$ in both the $x$ and $y$ coordinates. In configuration \#4, the sensor gain $g$ is perturbed to vary between $[1-\Delta g, 1+\Delta g]$ with $\Delta g=0.5$.

Each experiment will be repeated 500 times using a cost function evaluated with mode 0 setting and another 500 times with a cost function evaluated, using the mode 1 setting. The mean and the STD of the estimation error on $x$ and $y$-axis are summarized in Figure 5.

Averaged over the four different parameter settings listed in Table 1, the mean and variance of each method in both $x$ and $y$ directions are listed in Table 2. Using T-test, it is found that the differences in terms of the mean values of the position estimation errors among the four different search methods are statistically insignificant. Hence, despite large number of cost function evaluations, the ES method does not offer significant benefit in terms of improving source localization accuracy. Of course, this conclusion is conditioned on the practice implemented in this experiment to conduct initial coarse-grained ES (at 5 meters resolution) before commencing the three local search algorithms, namely,
TABLE 2: Mean and variance of four different optimization methods, averaged over four test conditions.

\begin{tabular}{lcccc}
\hline & Mean- $x$ & Var- $x$ & Mean- $y$ & Var- $y$ \\
\hline ES & 0.093925 & 5.939293 & -0.042100 & 6.466883 \\
MR & 0.082425 & 6.242463 & -0.030850 & 6.671392 \\
DS & 0.086488 & 8.287125 & -0.053988 & 8.492783 \\
GD & 0.074825 & 3.145920 & 0.029475 & 3.343724 \\
\hline
\end{tabular}

MR, DS, and GD. Without this initial ES, these methods may be trapped in a local minimum solution that yields much larger position estimation error.

The simulation results can also be used to compare the effectiveness of evaluating the cost function using mode \#0 (using minimum number of $N-1$ energy ratios) versus mode \#1 (using maximum number of $N(N-1) / 2$ energy ratios) configurations. The results are listed in Table 3.

When the gain variation results are included, mode \#1 performs worse than mode $\# 0$. This is because the erroneous energy reading will be used to compute $N-1$ energy ratios in the mode \#1 configuration and only 1 energy ratio for the mode \#0 configuration. Hence the same amount of error on a single sensor reading will have a bigger impact in mode \#1 than mode $\# 0$. However, excluding the gain variation factor, in general, mode \#1 performs much better than mode $\# 0$. This result indicates that gain calibration of microphone is essential to the success of the energy-based source localization method presented in this paper. This point is also clearly illustrated in Figure 5. 
TABle 3: Comparison between mode \#0 and mode \#1 results, averaged over all the four different methods, with different parameter variations.

\begin{tabular}{lcccc}
\hline Include $d_{g}$ & Mean- $x$ & Var- $x$ & Mean- $y$ & Var- $y$ \\
\hline Mode-0 & 0.081675 & 4.087787 & 0.0216 & 4.115982 \\
Mode- 1 & 0.091267 & 9.244179 & -0.10443 & 10.0473 \\
\hline Exclude $d_{g}$ & Mean- $x$ & Var- $x$ & Mean- $y$ & Var- $y$ \\
\hline Mode-0 & 0.044933 & 2.959709 & 0.048542 & 2.694365 \\
Mode- 1 & 0.001889 & 0.573453 & -0.00394 & 0.107678 \\
\hline
\end{tabular}

\subsection{Sensitivity analysis to parameter perturbations}

In the previous section, we compared the performance of four different search methods. In this section, we will investigate how the accuracy of the energy-based source localization method will be affected by inaccurate measurements of parameters or the presence of noise.

\subsubsection{Factors affecting localization accuracy}

(a) Energy decay exponent $\alpha$. Although we have conducted preliminary experiment and determined that the acoustic energy decay exponent $\alpha$ is approximately 2 . However, this result is obtained using a point, omnidirectional sound source in a favorable environment where the breeze is gentle and the temperature is mild. It is likely that this parameter may be varied at different situations. Thus, it is important to understand how sensitive the localization result will be with respect to inaccurate estimate of the value of $\alpha$.

(b) Sensor coordinate measurement $r_{i}$. Sensor coordinates can be obtained using on board global positioning system (GPS) readings if such a device is available. However, highly accurate sensor location measurements would require longterm averaging of GPS readings and may consume extensive battery power. It is necessary to study what will be the impact of sensor location inaccuracy on the accuracy of energybased target localization.

(c) Acoustic sensor gain measurement $g_{i}$. Not all acoustic sensors are identical. Different sensors may exhibit different gain characteristics. Thus, it is crucial to calibrate the gain factor of individual acoustic sensors. It is also important to gauge the effect of gain calibration error on the target localization accuracy.

(d) Acoustic energy measurement-signal-to-noise ratio $(S N R)$. As discussed earlier, the acoustic energy is usually averaged over a predefined time window as the sum of squares of acoustic time series data (with mean subtracted). Energy readings estimated this way may contain the energy of the background noise. Suppose that the noise time series is modeled as a white Gaussian random process, its energy should have a $\chi^{2}$ distribution. However, if the number of time samples within each time window is sufficiently large, using central limit theorem, the noise energy can be modeled with an equivalent Gaussian random process. Note that although the noise energy level is likely to be the same over neighboring sensor nodes, the source energy measured at different sen-
TABle 4: Parameter settings for the experiments to examine the localization to perturbation.

\begin{tabular}{cclllc}
\hline Configuration \# & Grid size & $\Delta \alpha$ & $\Delta r$ & $\Delta g$ & SNR $(\mathrm{dB})$ \\
\hline 1 & $1 \times 1$ & 0 & 0 & 0 & $\infty$ \\
2 & $5 \times 5$ & 0 & 0 & 0 & $\infty$ \\
3 & $10 \times 10$ & 0 & 0 & 0 & $\infty$ \\
4 & $1 \times 1$ & 0.2 & 0 & 0 & $\infty$ \\
5 & $1 \times 1$ & 0.5 & 0 & 0 & $\infty$ \\
6 & $1 \times 1$ & 1 & 0 & 0 & $\infty$ \\
7 & $1 \times 1$ & 0 & 0.5 & 0 & $\infty$ \\
8 & $1 \times 1$ & 0 & 1 & 0 & $\infty$ \\
9 & $1 \times 1$ & 0 & 5 & 0 & $\infty$ \\
10 & $1 \times 1$ & 0 & 0 & 0.2 & $\infty$ \\
11 & $1 \times 1$ & 0 & 0 & 0.5 & $\infty$ \\
12 & $1 \times 1$ & 0 & 0 & 1 & $\infty$ \\
13 & $1 \times 1$ & 0 & 0 & 0 & 20 \\
14 & $1 \times 1$ & 0 & 0 & 0 & 10 \\
15 & $1 \times 1$ & 0 & 0 & 0 & 0 \\
\hline
\end{tabular}

sor nodes are different according to the energy decay model. In fact, due to energy decay, the SNR reduces by a factor of $\left(a \log _{10}\left|r-r_{i}\right|\right)$ provided that the background noise energy levels at every sensor are the same. If $\alpha \approx 2$, this means $2 \mathrm{~dB}$ SNR reductions for every additional 10 meters distance. Hence, the SNR, measured at a sensor that is 50 meters away from the source, will be $10 \mathrm{~dB}$ less than the SNR measured at 1 meter from the same source.

\subsubsection{Simulation method}

In this experiment, 20 randomly located sensors are used to locate a randomly placed target. Both are located within a predefined sensor field. We use the ES algorithm to minimize the cost function. As listed in Table 4, 15 configurations are designed for this experiment. The first three configurations are designed to compare the effect of different grid size for ES. Three grid resolutions 1 meter, 5 meters, and 10 meters are used. The purpose of configurations \#4 to \#6 is to compare the algorithm sensitivity with respect to variations of exponential decaying factor $\alpha$. The actual value of $\alpha$ is randomly drawn from the interval $[\alpha-\Delta \alpha, \alpha+\Delta \alpha]$ with $\Delta \alpha=0.2,0.5$, and 1 . Configurations $\# 7$ to $\# 9$ are designed to compare the effect of inaccurate sensor locations measurement. Each sensor location vector $r$ is randomly perturbed as $r+\Delta r$ where $\Delta r=[\Delta x, \Delta y]$ and $\Delta x, \Delta y$ are both random variables uniformly distributed over an interval (in meters) $[-0.5,0.5],[-1,1]$, or $[-5,5]$. In configurations \#10 to \#12, we intend to examine the impacts of inaccuracy in acoustic sensor gain variation. The actual sensor gain is drawn randomly from a uniform distribution $[1-\Delta g, 1+\Delta g$ ]. Our aim in designing configurations $\# 13$ to $\# 15$ is to examine the effects of different SNRs. The energy variations in these configurations, specified in $\mathrm{dB}$, are measured at 1 meter away from the source. As we discussed earlier, the actual SNR at each sensor varies, depending on the relative distance to the 
TABLE 5: Mean (bias) and STD of simulation results using different grid sizes.

\begin{tabular}{|c|c|c|c|c|c|c|}
\hline \multirow{2}{*}{ Bias } & \multicolumn{3}{|c|}{$x$-coordinate } & \multicolumn{3}{|c|}{$y$-coordinate } \\
\hline & $1 \times 1$ grid & $5 \times 5$ grid & $10 \times 10$ grid & $1 \times 1$ grid & $5 \times 5$ grid & $10 \times 10$ grid \\
\hline$(20,19)$ & 0.041 & -0.0434 & 0.0649 & 0.0576 & 0.1755 & 0.1615 \\
\hline$(10,9)$ & 0.009 & 0.0216 & -0.0451 & 0.0396 & 0.2005 & 0.0915 \\
\hline$(5,4)$ & 0.01 & 0.0516 & 0.1149 & 0.0316 & 0.0755 & 0.0115 \\
\hline$(20,190)$ & -0.007 & 0.0216 & -0.1151 & 0.0176 & -0.0045 & -0.0285 \\
\hline$(10,45)$ & -0.006 & -0.0134 & -0.1151 & 0.0166 & -0.0395 & 0.0015 \\
\hline$(5,10)$ & -0.01 & 0.0366 & -0.0151 & 0.0146 & 0.0305 & -0.1285 \\
\hline \multirow{2}{*}{ STD } & \multicolumn{3}{|c|}{$x$-coordinate } & \multicolumn{3}{|c|}{$y$-coordinate } \\
\hline & $1 \times 1$ grid & $5 \times 5$ grid & $10 \times 10$ grid & $1 \times 1$ grid & $5 \times 5$ grid & $10 \times 10$ grid \\
\hline$(20,19)$ & 0.7792 & 2.7691 & 4.4097 & 0.7147 & 2.7548 & 4.2629 \\
\hline$(10,9)$ & 0.673 & 2.4047 & 4.0726 & 0.6453 & 2.418 & 4.0569 \\
\hline$(5,4)$ & 0.83 & 2.5052 & 4.0247 & 0.721 & 2.71 & 4.2094 \\
\hline$(20,190)$ & 0.3036 & 1.4664 & 3.0664 & 0.296 & 1.4916 & 2.9337 \\
\hline$(10,45)$ & 0.3054 & 1.5007 & 3.111 & 0.2978 & 1.5019 & 2.9642 \\
\hline$(5,10)$ & 0.3307 & 1.5853 & 3.3359 & 0.3151 & 1.5612 & 3.2578 \\
\hline
\end{tabular}

source. $\mathrm{SNR}=\infty$ implies that there is no noise, that is, $\varepsilon=0$. $\mathrm{SNR}=0$ means that the noise energy is equal to that of the source energy. The perturbations on $r, g$, and SNR are applied to all individual sensors.

As in the previous experiment, different numbers of sensors and numbers of energy ratios may affect the localization accuracy. To better understand their impact, we devised six different modes and denoted this combination, using a vector $(N, M)$, where $N=$ number of sensors used and $M=$ number of energy ratios used. These modes are $(20,19)$, $(10,9),(5,4),(20,190),(10,45)$, and $(5,10)$. In the first three modes, $M=N-1$. In the last three, $M=N(N-1) / 2$. For each configuration and each of the mode, 1000 independent simulations are performed and the mean and STD of the results in both $x$ and $y$ directions are computed for further analysis.

\subsubsection{Results and discussions}

(a) Different grid size (search resolution). The simulation results corresponding to configurations \#1 to \#3 are listed in Table 5.

The following two observations are worth noting.

(i) Bias-the energy-based source localization method yields unbiased estimate at each of the three grid sizes.

(ii) Variance-suppose that the target location is uniformly and randomly distributed within a grid, then the expected STD of position estimation error will be $\ell / \sqrt{12} \cong 0.2887 \ell$ at each $x$ - and $y$-direction. From Table 5, it is clear that when the maximum number of energy ratios are used, that is, $M=N(N-1) / 2$, the position estimation error will approximate this lower bound. On the other hand, when $M=N-1$, the variances are uniformly larger. This is more prominent when the grid size is small. Our conjecture is that the cost functions formed, using $N-1$ energy ratios does not, have the same global minimum as the cost function formed using $N(N-1) / 2$ energy ratios.

(b) Variation on $\alpha$-the results corresponding to configurations \#1, 4, 5, and 6 are listed in Table 6 .

Again, we make two observations on this table.

(i) Bias - the variation of the energy decay exponent $\alpha$ has little effect on the bias of the estimation error.

(ii) STD - the variations of $\alpha$ did impact the results when $M=N-1$. It seems that the more sensors are used, the larger the STD is. On the other hand, when $M=$ $N(N-1) / 2$, the variation of $\alpha$ as large as 1 , that is, the values of $\alpha$ varies between 1 and 3 , has little effect on the STD of the location estimation error. This is an important evidence to justify the use of a nominal value of $\alpha=2$ provided the maximum number of energy ratios is included in the cost function definition.

(c) Variations on sensor position error $r$ - the results are summarized in Table 7.

As in the previous cases, the sensor location errors will not impose any bias to the location estimates. What is different from the previous cases is that the STD of estimation errors seem to be similar using either $M=N-1$ or $M=N(N-1) / 2$ energy ratios.

(d) Variations on sensor gain $g$-the results are summarized in Table 8.

Consistent with the results obtained in the previous experiment, the energy-based source localization algorithm is quite sensitive to the error in gain calibration. In particular, in terms of STD, two important trends can be observed from Table 8 .

(i) More sensors give worse results. Apparently, more sensors with wrong gain factor will impact significantly 
TABLE 6: Mean and STD of position estimate errors due to variation of $\alpha$.

\begin{tabular}{|c|c|c|c|c|c|c|c|c|}
\hline \multirow{2}{*}{ Bias } & \multicolumn{4}{|c|}{$x$-coordinate } & \multicolumn{4}{|c|}{$y$-coordinate } \\
\hline & $\Delta \alpha=0$ & $\Delta \alpha=0.2$ & $\Delta \alpha=0.5$ & $\Delta \alpha=1$ & $\Delta \alpha=0$ & $\Delta \alpha=0.2$ & $\Delta \alpha=0.5$ & $\Delta \alpha=1$ \\
\hline$(20,19)$ & 0.041 & -0.07 & -0.0347 & -0.2275 & 0.0576 & 0.0279 & -0.0236 & -0.0062 \\
\hline$(10,9)$ & 0.009 & -0.086 & -0.0047 & -0.0875 & 0.0396 & 0.0189 & -0.0116 & -0.0082 \\
\hline$(5,4)$ & 0.01 & -0.055 & 0.0593 & -0.0395 & 0.0316 & 0.0229 & 0.0104 & -0.0252 \\
\hline$(20,190)$ & -0.007 & 0.004 & -0.0177 & 0.0075 & 0.0176 & 0.0039 & -0.0026 & -0.0072 \\
\hline$(10,45)$ & -0.006 & 0.003 & -0.0097 & 0.0035 & 0.0166 & 0.0099 & -0.0046 & -0.0002 \\
\hline$(5,10)$ & -0.01 & 0.004 & -0.0027 & 0.0145 & 0.0146 & 0.0129 & -0.0076 & -0.0002 \\
\hline \multirow{2}{*}{ STD } & \multicolumn{4}{|c|}{$x$-coordinate } & \multicolumn{4}{|c|}{$y$-coordinate } \\
\hline & $\Delta \alpha=0$ & $\Delta \alpha=0.2$ & $\Delta \alpha=0.5$ & $\Delta \alpha=1$ & $\Delta \alpha=0$ & $\Delta \alpha=0.2$ & $\Delta \alpha=0.5$ & $\Delta \alpha=1$ \\
\hline$(20,19)$ & 0.7792 & 0.8585 & 1.4971 & 2.9646 & 0.7147 & 0.9253 & 1.4706 & 2.9502 \\
\hline$(10,9)$ & 0.673 & 0.7729 & 1.1727 & 2.183 & 0.6453 & 0.7842 & 1.205 & 2.1486 \\
\hline$(5,4)$ & 0.83 & 0.7788 & 1.2808 & 2.1823 & 0.721 & 0.8247 & 1.4049 & 1.993 \\
\hline$(20,190)$ & 0.3036 & 0.3007 & 0.2841 & 0.2935 & 0.296 & 0.2993 & 0.2962 & 0.291 \\
\hline$(10,45)$ & 0.3054 & 0.3037 & 0.2833 & 0.2979 & 0.2978 & 0.2996 & 0.3007 & 0.2898 \\
\hline$(5,10)$ & 0.3307 & 0.3225 & 0.3273 & 0.3549 & 0.3151 & 0.3259 & 0.3276 & 0.3286 \\
\hline
\end{tabular}

TABLE 7: Mean and STD of source location estimation error due to different sensor location errors.

\begin{tabular}{|c|c|c|c|c|c|c|c|c|}
\hline \multirow{2}{*}{ Bias } & \multicolumn{4}{|c|}{$x$-coordinate } & \multicolumn{4}{|c|}{$y$-coordinate } \\
\hline & $d(r)=0$ & $d(r)=0.5$ & $d(r)=1$ & $d(r)=5$ & $d(r)=0$ & $d(r)=0.5$ & $d(r)=1$ & $d(r)=5$ \\
\hline$(20,19)$ & 0.041 & 0.0245 & -0.0088 & -0.0186 & 0.0576 & 0.0136 & -0.0121 & 0.1262 \\
\hline$(10,9)$ & 0.009 & 0.0195 & -0.0848 & 0.0074 & 0.0396 & 0.0586 & 0.0149 & 0.1362 \\
\hline$(5,4)$ & 0.01 & 0.0505 & -0.0538 & 0.0274 & 0.0316 & 0.0546 & -0.0031 & -0.0088 \\
\hline$(20,190)$ & -0.007 & 0.0005 & 0.0232 & -0.0876 & 0.0176 & -0.0064 & 0.0109 & 0.0852 \\
\hline$(10,45)$ & -0.006 & 0.0185 & 0.0132 & 0.0154 & 0.0166 & -0.0074 & 0.0829 & 0.0092 \\
\hline$(5,10)$ & -0.01 & 0.0235 & -0.0378 & 0.1484 & 0.0146 & 0.0176 & -0.0211 & -0.0978 \\
\hline \multirow{2}{*}{ STD } & \multicolumn{4}{|c|}{$x$-coordinate } & \multicolumn{4}{|c|}{$y$-coordinate } \\
\hline & $d(r)=0$ & $d(r)=0.5$ & $d(r)=1$ & $d(r)=5$ & $d(r)=0$ & $d(r)=0.5$ & $d(r)=1$ & $d(r)=5$ \\
\hline$(20,19)$ & 0.7792 & 0.873 & 1.0054 & 3.5845 & 0.7147 & 0.842 & 1.0074 & 3.5751 \\
\hline$(10,9)$ & 0.673 & 0.8418 & 1.0797 & 3.7525 & 0.6453 & 0.8391 & 1.0405 & 3.594 \\
\hline$(5,4)$ & 0.83 & 1.061 & 1.6229 & 4.093 & 0.721 & 1.1529 & 1.6245 & 4.4003 \\
\hline$(20,190)$ & 0.3036 & 0.3672 & 0.5774 & 4.8538 & 0.296 & 0.3664 & 0.5541 & 4.9591 \\
\hline$(10,45)$ & 0.3054 & 0.4718 & 0.8653 & 4.0243 & 0.2978 & 0.4458 & 0.8016 & 3.8904 \\
\hline$(5,10)$ & 0.3307 & 0.8271 & 1.5717 & 3.9941 & 0.3151 & 0.8392 & 1.7502 & 4.2185 \\
\hline
\end{tabular}

the shape of the cost function and therefore the location of its minimum.

(ii) Using $M=N-1$ or $M=N(N-1) / 2$ yields approximately the same quality of the results. The favor is slightly tilted toward the former. However, the difference is not statistically significant.

The key lesson learned from these three configurations is that sensor gain calibration is crucial to the success of this algorithm. Hence, each sensor should be calibrated before deployment in the field.

Table 9
The effect of additive background noise is similar to that of sensor gain perturbation: both will affect the accuracy of energy measurements at each sensor. From Table 9, one observes that

(i) the more sensors are used, the larger the STD. Apparently, the energy estimation errors do not cancel each other when more sensor readings are used.

(ii) other than $\mathrm{SNR}=\infty$, the two modes $M=N-1$ and $M=N(N-1) / 2$ yield approximately the same standard deviation. The differences increase when more sensors are being used.

We must note that for practical vehicle target, the SNR at 
TABLE 8: Mean and STD of localization error for different sensor gain values.

\begin{tabular}{|c|c|c|c|c|c|c|c|c|}
\hline \multirow{2}{*}{ Mean } & \multicolumn{4}{|c|}{$x$-coordinate } & \multicolumn{4}{|c|}{$y$-coordinate } \\
\hline & $d(g)=0$ & $d(g)=0.2$ & $d(g)=0.5$ & $d(g)=1$ & $d(g)=0$ & $d(g)=0.2$ & $d(g)=0.5$ & $d(g)=1$ \\
\hline$(20,19)$ & 0.041 & 0.0869 & 0.0152 & 0.0727 & 0.0576 & 0.0148 & 0.0143 & 0.3178 \\
\hline$(10,9)$ & 0.009 & 0.0369 & -0.0058 & 0.0977 & 0.0396 & 0.0268 & 0.0043 & 0.1988 \\
\hline$(5,4)$ & 0.01 & 0.0619 & 0.0512 & -0.0553 & 0.0316 & -0.0582 & 0.0253 & 0.1958 \\
\hline$(20,190)$ & -0.007 & 0.1429 & 0.1452 & 0.0217 & 0.0176 & 0.0168 & 0.2463 & 0.5738 \\
\hline$(10,45)$ & -0.006 & 0.0519 & 0.1922 & 0.1137 & 0.0166 & 0.0808 & 0.1133 & 0.2618 \\
\hline$(5,10)$ & -0.01 & 0.0489 & 0.0292 & 0.0067 & 0.0146 & -0.0022 & 0.0233 & 0.2008 \\
\hline \multirow{2}{*}{ STD } & \multicolumn{4}{|c|}{$x$-coordinate } & \multicolumn{4}{|c|}{$y$-coordinate } \\
\hline & $d(g)=0$ & $d(g)=0.2$ & $d(g)=0.5$ & $d(g)=1$ & $d(g)=0$ & $d(g)=0.2$ & $d(g)=0.5$ & $d(g)=1$ \\
\hline$(20,19)$ & 0.7792 & 1.3112 & 0.852 & 9.0153 & 0.7147 & 1.2086 & 3.5631 & 9.1049 \\
\hline$(10,9)$ & 0.673 & 1.5515 & 3.2465 & 6.5942 & 0.6453 & 1.5616 & 3.1859 & 6.3942 \\
\hline$(5,4)$ & 0.83 & 2.0879 & 3.213 & 4.9774 & 0.721 & 1.9148 & 3.2588 & 4.992 \\
\hline$(20,190)$ & 0.3036 & 2.9657 & 7.4987 & 10.2708 & 0.296 & 2.7917 & 7.0885 & 10.0506 \\
\hline$(10,45)$ & 0.3054 & 2.3371 & 4.2964 & 6.0754 & 0.2978 & 2.1831 & 4.1489 & 5.9161 \\
\hline$(5,10)$ & 0.3307 & 2.2247 & 3.3303 & 4.526 & 0.3151 & 2.1157 & 3.3239 & 4.3258 \\
\hline
\end{tabular}

TABLE 9: Mean and STD of position estimation error due to background noise.

\begin{tabular}{|c|c|c|c|c|c|c|c|c|}
\hline \multirow{2}{*}{ Mean } & \multicolumn{4}{|c|}{$x$-coordinate } & \multicolumn{4}{|c|}{$y$-coordinate } \\
\hline & $\mathrm{SNR}=\infty$ & $\mathrm{SNR}=20 \mathrm{~dB}$ & $\mathrm{SNR}=10 \mathrm{~dB}$ & $\mathrm{SNR}=0 \mathrm{~dB}$ & $\mathrm{SNR}=\infty$ & $\mathrm{SNR}=20 \mathrm{~dB}$ & $\mathrm{SNR}=10 \mathrm{~dB}$ & $\mathrm{SNR}=0 \mathrm{~dB}$ \\
\hline$(20,19)$ & 0.041 & -0.1776 & 0.1094 & -0.082 & 0.0576 & 0.2093 & 0.0268 & 0.5168 \\
\hline$(10,9)$ & 0.009 & -0.2166 & 0.1604 & 0.08 & 0.0396 & 0.1833 & 0.0228 & 0.2688 \\
\hline$(5,4)$ & 0.01 & -0.1146 & 0.1784 & -0.035 & 0.0316 & 0.0243 & -0.1152 & 0.1878 \\
\hline$(20,190)$ & -0.007 & -0.6146 & 0.4334 & -0.202 & 0.0176 & 0.2893 & -0.0312 & 0.7268 \\
\hline$(10,45)$ & -0.006 & -0.1546 & 0.3234 & -0.111 & 0.0166 & 0.3033 & -0.0822 & 0.3788 \\
\hline$(5,10)$ & -0.01 & -0.0896 & 0.1784 & -0.103 & 0.0146 & -0.0397 & -0.0312 & 0.1738 \\
\hline \multirow{2}{*}{ STD } & \multicolumn{4}{|c|}{$x$-coordinate } & \multicolumn{4}{|c|}{$y$-coordinate } \\
\hline & $\mathrm{SNR}=\infty$ & $\mathrm{SNR}=20 \mathrm{~dB}$ & $\mathrm{SNR}=10 \mathrm{~dB}$ & $\mathrm{SNR}=0 \mathrm{~dB}$ & $\mathrm{SNR}=\infty$ & $\mathrm{SNR}=20 \mathrm{~dB}$ & $\mathrm{SNR}=10 \mathrm{~dB}$ & $\mathrm{SNR}=0 \mathrm{~dB}$ \\
\hline$(20,19)$ & 0.7792 & 5.6954 & 8.1004 & 9.837 & 0.7147 & 5.5849 & 7.5979 & 9.4387 \\
\hline$(10,9)$ & 0.673 & 3.8446 & 5.6287 & 6.7225 & 0.6453 & 3.9481 & 5.2083 & 6.4093 \\
\hline$(5,4)$ & 0.83 & 2.8921 & 4.4332 & 5.2167 & 0.721 & 3.1086 & 3.9147 & 5.2041 \\
\hline$(20,190)$ & 0.3036 & 10.9375 & 12.6961 & 13.5552 & 0.296 & 10.9602 & 12.4986 & 13.3184 \\
\hline$(10,45)$ & 0.3054 & 5.1254 & 6.5798 & 7.2908 & 0.2978 & 5.2017 & 6.5202 & 7.1348 \\
\hline$(5,10)$ & 0.3307 & 3.0126 & 4.5768 & 5.0284 & 0.3151 & 3.1965 & 4.1028 & 4.9295 \\
\hline
\end{tabular}

the source is often much higher than $40 \mathrm{~dB}$. The condition of $0 \mathrm{~dB}$ or worse may occur when strong wind directly blowing into a microphone without wind-damper protection, or the microphone is hit by blowing debris or similar interferences.

\subsubsection{Discussion}

Based on the above two experiments, one may deduce the following guidelines for the proper implementation of the energy-based acoustic source localization algorithm:

(i) proper definition of the sensor field where the potential target localization will lie; (ii) careful calibration of sensor gain factor;

(iii) use one of the fast search algorithm MR, GD, or simplex DS method after first conducting a coarse-grained ES within the sensor field;

(iv) using few reliable energy readings from a few sensor is preferred to using many unreliable energy readings from more sensors. If one may assess the accuracy of individual energy reading, it will be possible to prune out unreliable sensor readings to enhance the overall localization accuracy;

(v) using more energy ratios (i.e., $M=N(N-1) / 2$ ) often yield more reliable results. 

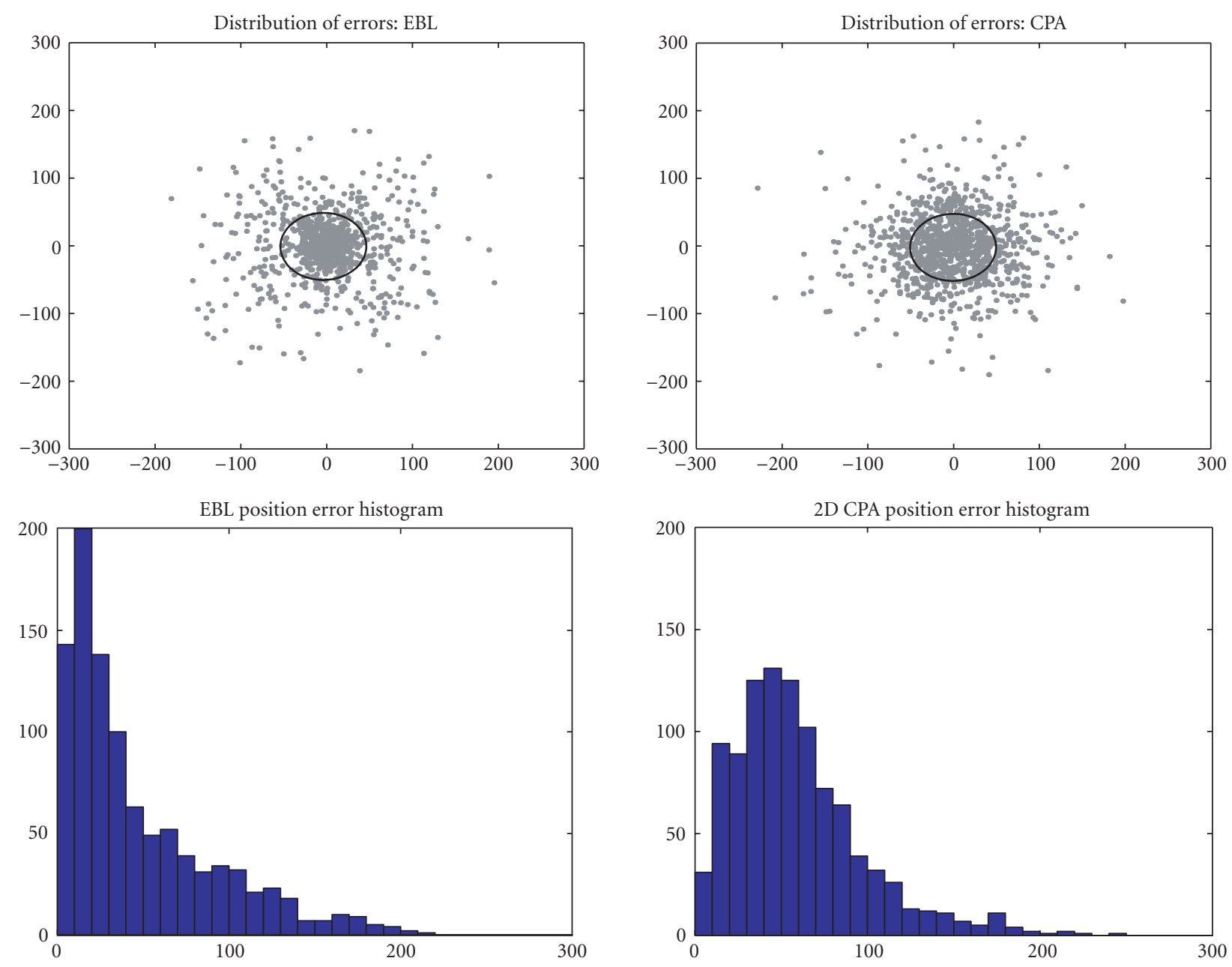

FIgURE 6: Comparison between EBL and CPA localization method.

\subsection{Comparison with other acoustic localization methods}

The energy-based single acoustic source localization method presented above differs from other existing method in a number of important aspects, as follows.

(1) Target positions are estimated at constant time interval-with the CPA-based approach, a new target location is obtained only when the moving target passes through another sensor. If the target stopped and remain stationary for a period of time, no additional CPA detection will be made. With energy-based source localization method, as long as the target continue to emit acoustic energy, its location will be estimated on a regular time interval, even when the target vehicle is idling and remain stationary. This significantly simplifies the task of the tracking algorithm.

(2) Energy-based method reduces communication requirements over wireless channels, and hence conserves power-energy is a scalar quantity that is computed over a number of data samples. The frequency of how often an energy reading is computed can be easily adjusted to meet the performance requirement and communication bandwidth as well as energy consumption constraints. Time delay-based localization methods will require accurate estimate of relative time delays (or phase difference in frequency domain) between different sensors. Hence, they may require more raw data samples or corresponding frequency components to be exchanged between sensor nodes.

We conducted a preliminary experiment comparing the proposal EBL algorithm with the 2D CPA algorithm. A sensor field of 300 meters by 300 meters is deployed with eight acoustic sensors at random locations. The target location is also randomly chosen within the same sensor field. Both sensor locations and target locations are drawn from a uniform distribution. The measured sensor locations, however, are assumed to suffer a measurement error that is uniformly distributed over $[-0.5,0.5]$ meters. The acoustic sensor gain $g$ is assumed to vary between 0.6 and 1.2 compared to a calibrated value of 1 . Each sensor is also subject to a $20 \mathrm{~dB}$ 
TABLE 10: Mean and STD of the estimation error.

\begin{tabular}{lcc|cc}
\hline & \multicolumn{2}{c|}{ EBL } & \multicolumn{2}{c}{ CPA } \\
\hline Mean value & -0.14873 & -0.60246 & 0.41733 & -0.72433 \\
STD & 49.0514 & 46.5717 & 48.292 & 53.8862 \\
\hline
\end{tabular}

additive Gaussian random noise with zero mean. The source energy level is fixed at a value of 1000 .

For the 2D CPA method, the measured sensor location corresponding to the sensor receiving maximum acoustic energy will be used as an estimate of the target location. For the EBL method, a search grid of 10 meters, each side will be used to enable an ES. The experiment contains 1000 independent trials. In each trial, the sensor locations, the target location, the perturbations on sensor location measurement, sensor gain variation, and additive noise are generated according to the specified distribution.

The mean and STDs of the target position estimation errors of these two methods are listed in Table 10.

The results are summarized in Figure 6. The ellipses in the top row specify the covariance matrices of these errors with each grey dot representing error incurred in a particular try. The histograms of the magnitudes of the position estimation errors are depicted at the bottom row.

\section{DISCUSSION AND CONCLUSION}

In this paper, we have presented the energy-based source localization algorithm, and derived theoretical results on the number of sensors required to yield a unique location estimate. We have also conducted extensive simulation to compare different search algorithms and to study the parameter sensitivity characteristics of this proposed algorithm.

An implicit advantage of this proposed algorithm is its simplicity: only acoustic energy measured during a specific period is needed. However, this simplicity also implies many practical difficulties that need to be mitigated. In particular, we note that the microphone gain calibration and SNR estimation are two key factors that affect the accuracy of this proposed algorithm.

Currently, we are working to apply this algorithm to real data obtained in the test ground. We are also studying potential extension of this algorithm to localize more than a single target within the sensor field.

\section{ACKNOWLEDGEMENTS}

This work was partly supported by DARPA under Grant no. F 30602-00-2-0555. The authors would like to thank Professors A. Sayeed, P. Ramanathan, K. Saluja at UW-Madison, Prof. K. Yao, and Dr. R. E. Hudson at University of California, Los Angles, for helpful discussions. The acoustic energy decay profile experiment is conducted with the assistance from UW-Madison SensIT team graduate students K. C. Wang, T. Chin, T. Clouquerur, V. Phipatanasuphcorn, A. Ashraf,
A. D'Costa, and M. Duarte. The authors would also like to extend their gratitude to the anonymous reviewers for their very constructive and helpful comments. In particular, Sections 3.6, 4.4, and 5.3 are added upon their suggestions.

\section{REFERENCES}

[1] D. Estrin, L. Girod, G. Pottie, and M. Srivastava, "Instrumenting the world with wireless sensor networks," in Proc. IEEE Int. Conf. Acoustics, Speech, Signal Processing, Salt Lake City, Utah, USA, May 2001.

[2] C. Savarese, J. M. Rabaey, and J. Beutel, "Locationing in distributed Ad-hoc wireless sensor networks," in Proc. IEEE Int. Conf. Acoustics, Speech, Signal Processing, Salt Lake City, Utah, USA, May 2001.

[3] N. L. Owsley, "Sonar array processing," in Array Signal Processing, S. Haykin, Ed., pp. 115-193, Prentice-Hall, Englewood Cliffs, NJ, USA, 1985.

[4] A. Tolstoy, Matched Field Processing for Underwater Acoustics, World Scientific Publications, Singapore, 1993.

[5] M. S. Brandstein, J. E. Adcock, and H. F. Silverman, "A localization-error-based method for microphone-array design," in Proc. IEEE Int. Conf. Acoustics, Speech, Signal Processing, Atlanta, Ga, USA, May 1996.

[6] M. S. Brandstein, J. E. Adcock, and H. F. Silverman, "A closedform location estimator for use with room environment microphone arrays," IEEE Trans. Speech, and Audio Processing, vol. 5, no. 1, pp. 45-50, 1997.

[7] J. Huang, N. Ohnishi, and N. Sugie, "Sound localization in reverberant environment based on the model of the precedence effect," IEEE Trans. Instrumentation and Measurement, vol. 46, no. 4, pp. 842-846, 1997.

[8] M. Omologo and P. Svaizer, "Acoustic source location in noisy and reverberant environment using CSP analysis," in Proc. IEEE Int. Conf. Acoustics, Speech, Signal Processing, Atlanta, Ga, USA, May 1996.

[9] K. Yao, R. E. Hudson, C. W. Reed, D. Chen, and F. Lorenzelli, "Blind beamforming on a randomly distributed sensor array system," IEEE Journal on Selected Areas in Communications, vol. 16, no. 8, pp. 1555-1567, 1998.

[10] C. W. Reed, R. Hudson, and K. Yao, "Direct joint source localization and propagation speed estimation," in Proc. IEEE Int. Conf. Acoustics, Speech, Signal Processing, Phoenix, Ariz, USA, May 1999.

[11] S. Haykin, J. H. Justice, N. L. Owsley, J. L. Yen, and A. C. Kak, Array Signal Processing, Prentice-Hall, Englewood Cliffs, NJ, USA, 1985.

[12] G. C. Carter, Coherence and Time Delay Estimation, IEEE Press, Mill Road Edison, NJ, USA, 1993.

[13] "Special issue on time-delay estimation," IEEE Trans. Acoustics, Speech, and Signal Processing, vol. 29, June 1981.

[14] J. O. Smith and J. S. Abel, "Closed-form least-squares location estimation from range-difference measurements," IEEE Trans. Acoustics, Speech, and Signal Processing, vol. 35, no. 12, pp. 1661-1669, 1987.

[15] L. Kinsler, A. Frey, A. Coppens, and J. Sanders, Fundamentals of Acoustics, John Wiley and Sons, New York, NY, USA, 1982.

[16] K. Srodecki, "Evaluation of the reverberation decay quality in rooms using the autocorrelation function and the cepstrum analysis," Acustica, vol. 80, no. 3, pp. 216-225, 1994.

[17] Y. L. Li, M. J. White, and S. J. Franke, "New fast field programs for anisotropic sound propagation through an atmosphere with a wind velocity profile," Journal of the Acoustical Society of America, vol. 95, pp. 718-726, 1994. 
[18] E. M. Salomons, "Downwind propagation of sound in an atmosphere with a realistic sound-speed profile: a semianalytical ray model," Journal of the Acoustical Society of America, vol. 95, no. 5, pp. 2425-2436, 1994.

[19] T. Watanabe and S. Yamada, "Sound attenuation through absorption by vegetation," Journal of the Acoustical Society of Japan, vol. 17, pp. 175-182, 1996.

[20] J. C. Chen, R. E. Hudson, and K. Yao, "A maximum likelihood parametric approach to source localization," in Proc. IEEE Int. Conf. Acoustics, Speech, Signal Processing, Salt Lake City, Utah, USA, May 2001.

[21] J. O. Smith and J. S. Abel, "The spherical interpolation method for closed-form passing source localization using range difference measurements," in Proc. IEEE Int. Conf. Acoustics, Speech, Signal Processing, Dallas, Tex, USA, 1987.

[22] R. E. Schwartz, "Minimax CFAR detection in additive Gaussian noise of unknown covariance," IEEE Transactions on Information Theory, vol. 15, pp. 722-725, 1969.

[23] J. Dabkowski Jr., "CFAR operation in non-homogeneous and point clutter," in Proc. National Electronics Conference, 1970.

Dan Li received the B.S.E.E. degree from Tsinghua University, Beijing, China, in 1996; the M.S. degree in biomedical engineering from the University of Kentucky in 1999; and the M.S.E.E. degree from the University of Wisconsin-Madison in 2001. He is currently a Researcher and Development Engineer at the Guidant Corporation, St. Paul, Minn, USA. His research interests are in algorithm development and applications of DSP and statistical signal processing.

Yu Hen $\mathbf{H u}$ received the B.S.E.E. degree from National Taiwan University, Taiwan, in 1976. He received the M.S. and Ph.D. degrees both in electrical engineering from University of Southern California, Los Angeles, Calif in 1980 and 1982, respectively. Currently, he is a Professor at the Electrical and Computer Engineering Department of the University of Wisconsin-Madison, Wis,

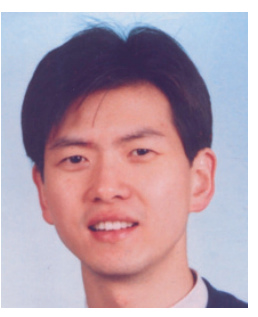
USA. Previously, he has been with the Elec-

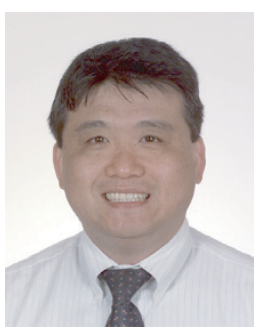
trical Engineering Department of the Southern Methodist University, Dallas, Tex, USA. Dr. Hu's research interests include multimedia signal processing, design methodology and implementation of signal processing algorithms and systems, sensor network and distributive signal processing algorithms, and neural network signal processing. He published more than 200 journal and conference papers and edited two books: Programmable Digital Signal Processors and Handbook of Neural Network Signal Processing. Dr. Hu is a Fellow of IEEE. He served as Associate Editor for IEEE Transactions on Signal Processing, IEEE Signal Processing Letters, Journal of VLSI Signal Processing, EURASIP Journal on Applied Signal Processing. He served as Secretary of IEEE signal processing society, board of governors of IEEE neural network council, Chair of IEEE signal processing society, and neural network signal processing technical committee. 